\title{
Code Phase Assignment DS/CDMA Communications for Indoor Wireless Rician Multipath Fading Channels
}

\author{
Johnny Yat-Hong Sung \\ yhjsung@hkueee.hku.hk
}

\author{
Kun-Wah Yip \\ kwyip@hkueee.hku.hk
}

\author{
Tung-Sang Ng \\ tsng@hkueee.hku.hk
}

Department of Electrical and Electronic Engineering,
The University of Hong Kong, Pokfulam Road, Hong Kong

Tel.: (852) 2857-8406 Fax.: (852) 2559-8738

\begin{abstract}
The technique of using a single basic signature sequence for the DS/CDMA system was investigated. All users have their sequences differed by cyclic phase shifts from a basic signature sequence. It is called Code Phase Assignment (CPA) [1]. In [1], it has been shown that the CPA DS/CDMA technique yielded better performance over conventional DS/CDMA communications by substantial reduction of the multiple access interference (MAI) and the multipath interference (MI) for a simplified channel model. This paper further investigates its performance in the indoor Rician multipath fading channel. Comparison with conventional DS/CDMA system will be made and the effect on the change of system parameters will be investigated. Higher multiple access capacity over conventional DS/CDMA will be demonstrated. Lastly, CPA could reduce the effort of exhaustive search for sequences which makes it suitable for indoor applications.
\end{abstract}

\section{INTRODUCTION}

There has been considerable interest in indoor wireless communications using DS/CDMA techniques [2]-[5] because of the ability in improving the performance through exploiting the multipath diversity. However, the performance of asynchronous DS/CDMA degrades considerably in the presence of MAI. To combat the adverse effects due to MAI, the CPA technique has been proposed for indoor wireless communications using DS/CDMA [1]. In CPA, signal transmission of all users are approximately synchronized and cross-correlation optimized cyclic codes are used for all signature sequences. These cyclic codes are generated from a basic sequence with different numbers of cyclic phase shifts. A basic sequence with good autocorrelation properties is selected in order to yield cyclic codes that are optimized in crosscorrelation properties. The CPA is aimed at (i) improving the error performance by reducing MAI and MI, (ii) reducing the effort in searching for optimized signature sequences, and (iii) providing higher multiple access capacity.

In this paper, we shall concentrate on comparing the error performance of CPA DS/CDMA and conventional DS/CDMA in the indoor environment. The indoor radio channel is modeled as a multipath Rician fading channel. Comparison of results is based on the following aspects: (i) different choices of signature sequences for the conventional DS/CDMA system. including m-sequences and Gold codes, (ii) the code length, (iii) the number of users, and (iv) the delay spread of the channel.

The paper is organized as follows. Section I is the introduction. Section II describes the CPA DS/CDMA system model. Section III presents the system performance and computational results which compares CPA and a conventional asynchronous DS/CDMA system under different conditions. Finally, conclusions are given in Section IV.

\section{CPA DS/CDMA SYSTEM MODEL}

\section{A. Transmitter Model}

The model considered here is a synchronous DS/CDMA system with $K$ users. Each user transmits a data waveform. The $k$-th $(1 \leq \mathrm{k} \leq \mathrm{K})$ user's data signal $b_{k}(t)$ is a sequence of positive and negative, unit pulses of duration $T_{b}$ that can be expressed as

$$
b_{k}(t)=\sum_{i=-\infty}^{\infty} b_{i}^{(k)} p_{T_{b}}\left(t-i T_{b}\right)
$$

where $b_{k, t} \in\{-1,+1\}, p_{\tau}(t)=1$ for $0 \leq \mathrm{t} \leq \tau$ and $p_{\tau}(t)=0$ otherwise.

Each user is assigned a signature sequence, $a_{k}(t)$ which has a period $T_{b}$, a length $\mathrm{N}$ and consists of positive and negative unit pulses. We assume that each chip $a_{j}^{(k)}$ in the periodic sequence has duration, $T_{c}$ so that there is one code period of $N$ chips $a_{0}^{(k)}, a_{1}^{(k)}, a_{2}^{(k)}, \ldots, a_{N-1}^{(k)}$ per data symbol $\left(N=T_{b} / T_{c}\right)$.

$$
\begin{aligned}
& a_{k}(t)=\sum_{j=-\infty}^{\infty} a_{j}^{(k)} p_{T_{c}}\left(t-j T_{c}\right) \\
& a_{j}^{(k)}=a_{j+x N}^{(k)} ; x \text { is an integer }
\end{aligned}
$$

where $a_{j}^{(k)} \in\{-1,+1\}$. It is proposed that all signature sequences $a_{1}(t), a_{2}(t), \ldots, a_{k}(t)$ are derived from a basic sequence. Each sequence is the cyclic phase shift replica from the basic one. For simplicity, it is assumed that a number of $m$ phase shift is assigned to any two consecutive signatures $a_{1}(t), a_{2}(t), \ldots, a_{k}(t)$. As a result, all sequences can be 
expressed in terms of the first signature $a_{1}(t)$ in addition with a tolerance, $n_{k} T_{c}$, in bit alignment during synchronization.

$$
\begin{aligned}
a_{k}(t) & =\sum_{j=-\infty}^{\infty} a_{j}^{(1)} p_{T_{e}}\left(t-j T_{c}-(k-1) m T_{c}-n_{k} T_{c}\right) \\
& =a_{1}\left(t-(k-1) m T_{c}-n_{k} T_{c}\right)
\end{aligned}
$$

For simplicity of calculation, the system is assumed to be perfectly synchronized and thus $n_{k} T_{\mathrm{c}}=0$. The transmitted $k$-th user signal can be denoted as

$$
s_{k}(t)=\sqrt{2 P} a_{k}(t) b_{k}(t) \cos \left(2 \pi f_{c} t+\theta_{k}\right)
$$

where $P$ represents the common signal power, $f_{c}$ represents the common carrier frequency and $\theta_{k}$ is the carrier phase which is uniformly distributed in $[0,2 \pi]$.

\section{B. Channel Model}

In the last tens of years, there are a lot of propagation measurements done to characterize the channel in the indoor environment. Measurements performed in several factories [6][8] shows that the wide-band channel can be modeled as Rician fading channel. The Rician distribution occurs when a strong path exists in addition to the low level scattered paths. The strong component may be a line-of-sight (LOS) path or a path that goes through much less attenuation compared to other arriving components. The complex lowpass equivalent impulse response of the Rician fading channel for the link between the $k$-th user and the receiver is expressed as

$$
h_{k}(t)=\sum_{q=1}^{L} \beta_{k q} \delta\left(t-\tau_{k q}\right) e^{j \psi_{k q}}
$$

where we assume there are $L$ resolvable paths associated with each user and $L=\left\lfloor\frac{\tau_{m m s}}{T_{c}}\right\rfloor+1$ where $\tau_{\text {rms }}$ is the root mean square delay spread. $\beta_{k q}, \tau_{k q}$ and $\psi_{k q}$ are the gain, delay and phase of the $k$-th user in the $q$-th path respectively. It is assumed that these $K$ users suffer from independent multipath fading such that $\left\{\boldsymbol{\beta}_{k q}\right\},\left\{\tau_{k q}\right\},\left\{\psi_{k q}\right\}$ are statistically independent. $\delta(t)$ is the Dirac-delta function.

It is assumed that $\tau_{k q}$ and $\psi_{k q}$ are uniformly distributed on $\left[0, T_{b}\right]$ and $[0,2 \pi]$ respectively. The path gain is a random variable which follows the Rician distribution with the probability density function given by

$$
p_{\beta}(\beta)=\frac{\beta}{\sigma^{2}} e^{-\left(\frac{\beta^{2}+a^{2}}{2 \sigma^{2}}\right)} I_{0}\left(\frac{a \beta}{\sigma^{2}}\right) \quad \beta \geq 0
$$

where $E\left[\beta^{2}\right]=a^{2}\left(1+\frac{2 \sigma^{2}}{a^{2}}\right)$. The factor $H=\frac{2 \sigma^{2}}{a^{2}}$ is the power ratio of the scattered component to the direct component. The measurements from [6] show that $H$ varies from $2 \mathrm{~dB}$ to $7 \mathrm{~dB}$. In this paper, $H$ is a constant of $6.8 \mathrm{~dB} . I_{0}()$ is the zeroth order modified Bessel function of the first kind.

The received signal at the input of the receiver is given by

$$
\begin{aligned}
r(t)= & \sqrt{2 P} \sum_{q=1}^{L} \sum_{k=1}^{K} \beta_{k q} a_{k}\left(t-\tau_{k q}\right) b_{k}\left(t-\tau_{k q}\right) \cos \left(2 \pi f_{c} t+\phi_{k q}\right) \\
& +n(t)
\end{aligned}
$$

where $\phi_{k q}=\theta_{k}+\psi_{k q}-2 \pi f_{c} \tau_{k q}$ is assumed to be uniform distributed in $[0,2 \pi]$ and $n(t)$ is the white Gaussian noise which has two-sided power spectral density $N_{0} / 2$.

\section{Receiver Model}

The whole DS/CDMA system is under network control so that the receivers and the transmitters are synchronised. The receiver consists of a matched filter and PSK demodulators that the signal are matched filtered and coherently demodulated to the transmitted bits. Without loss of generality, user 1 is the reference user. The decision variable for the user 1 in the path $l$ is given by $\xi_{i}^{(1)}$.

$$
\begin{aligned}
\xi_{1}^{(1)}= & \int_{0}^{T} r(t) a_{1}(t) \cos \left(2 \pi f_{c} t\right) d t \\
= & \sqrt{\frac{P}{2}} T b_{0}^{(i)} \beta_{1 l}+\sqrt{\frac{P}{2}} \sum_{\substack{q=1 \\
q \neq 1}}^{L} \beta_{1 q} \cos \phi_{1 q}\left[b_{-1}^{(1)} R_{1,1}\left(\tau_{1 q}\right)+b_{0}^{(1)} \hat{R}_{1,1}\left(\tau_{1 q}\right)\right] \\
& +\sqrt{\frac{P}{2}} \sum_{k=2 q=1}^{K} \sum_{k q}^{L} \beta_{k q} \cos \phi_{k q}\left[b_{-1}^{(k)} R_{k, 1}\left(\tau_{k q}\right)+b_{l}^{(k)} \hat{R}_{k, 1}\left(\tau_{k q}\right)\right]+n_{1}
\end{aligned}
$$

where $n_{1}$ is the additional white Gaussian noise (AWGN), For $\lambda T_{c} \leq \tau \leq(\lambda+1) T_{c}$ and $0 \leq t \leq T_{b}, \quad R_{k, I}$ and $\hat{R}_{k, I}$ are the continuous-time partial cross-correlation functions[1] defined by

$$
\begin{aligned}
R_{k, 1}(\tau) & =\int_{0}^{\tau} a_{k}(t-\tau) a_{1}(t) d t \\
R_{k, 1}(\tau) & =C_{k, 1}(\lambda-N) T_{c} \\
& +\left[C_{k, 1}(\lambda+1-N)-C_{k, 1}(\lambda-N)\right]\left(\tau-\lambda T_{c}\right)
\end{aligned}
$$

and $\hat{R}_{k, 1}(\tau)=\int_{\tau}^{T_{k}} a_{k}(t-\tau) a_{1}(t) d t$

$$
\hat{R}_{k, 1}(\tau)=C_{k, 1}(\lambda) T_{c}+\left[C_{k, 1}(\lambda+1)-C_{k, 1}(\lambda)\right]\left(\tau-\lambda T_{c}\right)
$$

where the $C_{k, 1}$ is the discrete aperiodic cross-correlation function for the sequences $\left(a_{j}^{(k)}\right)$ and $\left(a_{i}^{(k)}\right)$ defined by

$$
C_{k, 1}(\lambda)=\left\{\begin{array}{cc}
\sum_{j=0}^{N-1-\lambda} a_{j}^{(k)} a_{j+\lambda}^{(1)}, & 0 \leq \lambda \leq N-1 \\
\sum_{j=0}^{N-1+\lambda} a_{j-\lambda}^{(k)} a_{j}^{(1)}, & 1-N \leq \lambda<0 \\
0, & |\lambda| \geq N
\end{array}\right.
$$

Without loss of gencrality, for the reference user and the reference path $\tau_{11}=0$ and $\phi_{11}=0$. Moreover, for a synchronized system, all users are synchronized to the corresponding starting phase at the receiver and it is assumed the time delays for the first path of each user $\tau_{k 1}=0$ for $k=2, \ldots, K$. 
It is found that the equation (8) consists of four parts. Accordingly, they are the desired data, the MI due to the multipath channel, MAI from other users and the white Gaussian noise term with the variance $\operatorname{Var}\left\{n_{1}\right\}=\frac{N_{a} T_{b}}{4}$.

\section{SYSTEM PERFORMANCE AND COMPUTATIONAL RESULTS}

The system performance is determined by the average bit error probability, $P_{v}$ for a user. An error occurs when $\xi_{l}^{(1)}<0 \mid b_{1}^{0}=1$ or $\xi_{l}^{(1)}>0 \mid b_{1}^{0}=-1$. The average error probability for time-invariant and Rician fading channels is simulated by computers. They are shown in figure 1-4. For all figures, the number of resolvable paths is $L=4$, the length of codes $\mathrm{N}=127$ unless otherwise specified. In this section, we will compare the performance of the conventional CDMA and the CPA CDMA.

1) Conventional CDMA and CPA CDMA: The bit error probability of a conventional CDMA system is shown in figure 1. It is found that as the number of users is added to a conventional system, the multiple access interference increases and the bit error probabilities decreases. The results are compared with figure 2. In figure 2, the CPA CDMA with 127 m-sequence is used. The performance is improved at higher $E_{b} / N_{o}$ ratio. The main reason is the reduction of multiple access interferences with other users. At higher $E_{b} / N_{0}$, it is observed that the conventional CDMA with $\mathrm{K}=20$ has similar error performance with $\mathrm{CPA}$ with $\mathrm{K}=42$.

2) Comparison of CPA CDMA with $m$-sequence and Gold codes: Figure 2 and figure 3 compares with different codes. The Gold codes used here are non-optimized. It is found that they both have similar error performances at less users. However, when more users are subscribed to the system, the system with $\mathrm{m}$-sequence shows better performance. In this speckle case, the system with $\mathrm{m}$-sequence for $\mathrm{K}=42$ has approximate performance with Gold codes for $\mathrm{K}=30$. It is shown the choice of an optimized signature sequences in CPA CDMA is critical to the system performance. The right choice of a signature sequences can increase the number of users in CDMA system.

3) Comparison of CPA CDMA with different delay spread: In figure 4, there are six sets of data. We try to compare how the change in parameters may affect our system. First, we want to compare the effect of delay spread on the bit error probabilities. The common code length is $N=127$. but the number of resolvable paths are $\mathrm{L}=2$ and 4 respectively. We can conclude that the degradation in performance occurs if there is an increased value of the data rate or delay spread that increases $\mathrm{L}$. It is observed that at higher $E_{b} / N_{o}$ ratio where the multiple access interferences and the self multipath interferences dominate, the bit error probabilities of the case with $L=2$ is about one-fifth of the case with $\mathrm{L}=4$ for $\mathrm{K}=10$ and 30 .
4) Comparison of CPA CDMA with different code lengths, $N$ : Another comparison in figure 4 is made on the different number of code length while the number of paths remain unchanged $(\mathrm{L}=2)$. The bit error is about 3 times less for the same number of users. Moreover, it is found that the curve $\mathrm{N}=127, \mathrm{~K}=10$ overlaps with $\mathrm{N}=255, \mathrm{~K}=30$. The doubling of the code length can treble the capacity under non-diversity condition.

\section{CONCLUSIONS}

In this paper, the bit error performance of CPA DS/CDMA is evaluated. It is shown to have better performance than conventional asynchronous DS/CDMA. Comparison of results includes the following: (i) different choices of signature sequences for the CPA DS/CDMA system, including $\mathrm{m}$ sequences and Gold codes, (ii) the number of users, and (iii) the delay spread of the channel, and (iv) the code length.

It has been shown that CPA DS/CDMA can improve the bit error performance by reducing the MAI and the MI. Moreover, the algorithm of CPA is simple and it can reduce the effort in searching for optimized signature sequences. CPA can have higher multiple access capacity under a suitable choice of the basic signature sequence, lower delay spread and with longer code length. Hence, CPA DS/CDMA can be used in a typical indoor wireless environment where the delay spread is small compared with other common multipath fading channels[9].

\section{ACKNOWLEDGMENT}

This work was supported by the Hong Kong Research Grant Council and by the CRCG of The University of Hong Kong.

\section{REFERENCES}

1. K.W.Yip and T.S. Ng, "Code Phase Assignment - A Technique for High-Capacity Indoor Mobile DS/CDMA Communications," Proceedings, IEEE/VTS 44th Vehicular Technology Conference, vol 3, pp.1586-1590, Jun. 1994.

2. M.B.Pursley, "Performance Evaluation for Phase-Coded Spread Spectrum Multiple-Access Communication-Part I: System Analysis," IEEE Transactions on Communications, vol. COM-25, pp.795-799, Aug. 1977.

3. Wu K.T. and Tsaur S.A.,"Error performance for diversity DS/CDMA communications in fading channels.", IEE Proc.-commun., vol141,No.5, pp.357-363, Oct. 1994.

4. R. Prasad H. S. Misser and A. Kegel, "Performance Evaluation of Direct-Sequence Spread Spectrum MultipleAccess for Indoor Wireless Communication in a Rician Fading Channel," IEEE Trans. Commun., vol. 43, No. 2/3/4, pp. 581-592, Feb./Mar./Apr. 1995.

5. J. Wang, M. Moeneclaey and L. B. Milstein, "DS/CDMA with Predetection Diversity for Indoor Radio 
Communications," IEEE Trans. Commun., vol. COM-40, pp.1929-1938, Feb./Mar./Apr. 1995.

6. Robert J.C. Bultitude, "Measurement, characterization and modeling of indoor $800 / 900 \mathrm{MHz}$ radio channels for digital communications,", IEEE Commun. Mag., vol. 25, No.6, pp. 5-12, Jun. 1987.

7. T.S. Rappaport and C.D. McGillem, "UHF fading in factories," IEEE J. Select. Areas Commun., vol SAC-7, pp. 40-48, Jan. 1989.

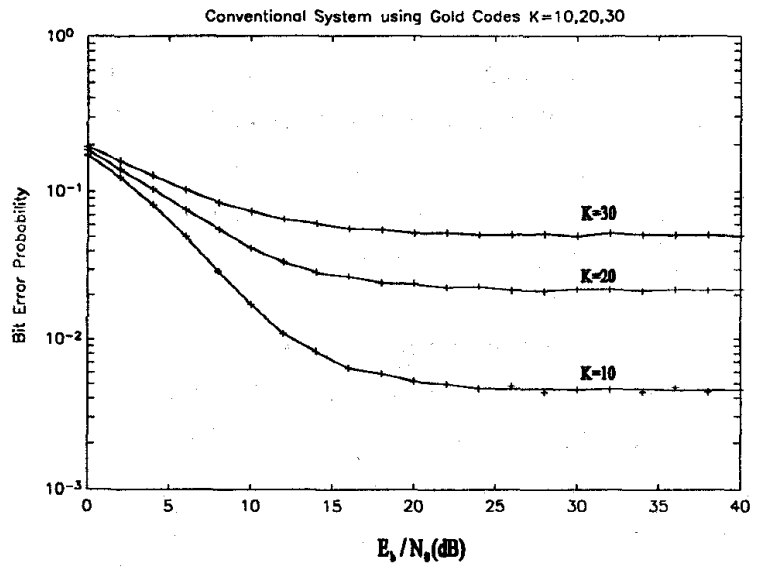

Figure 1. The performance of a conventional CDMA system over Rician multipath fading channels with different number of users using Gold codes $(\mathrm{N}=127)$.

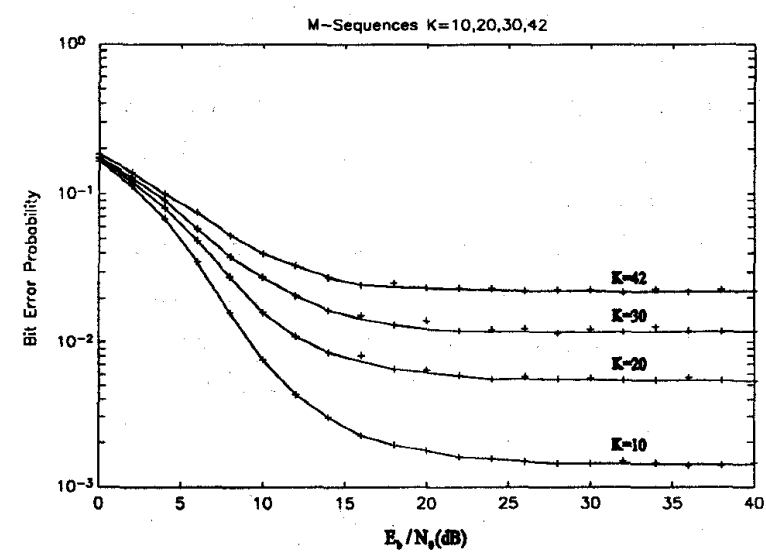

Figure 2. The performance of the CPA CDMA system over Rician multipath fading channels with different number of users $(K=10,20$ and 30$)$ using $m$-sequence $(N=127)$.
8. P.Yegani and C.D. McGillem,"A statistical model for lineof signal (LOS) factory radio channels." Proceedings, IEEE/VTS 39th Vehicular Technology Conference, pp. 496-503, May, 1989.

9. D. Molkdar, "Review on Radio Propagation into and within Buildings," IEE Proc.-H, vol. 138, pp. 61-73, Feb. 1991.

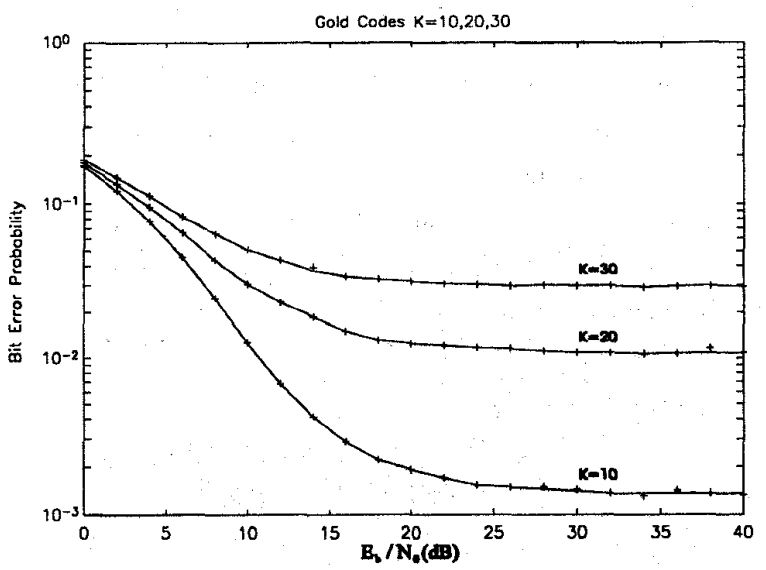

Figure 3. The performance of the CPA CDMA system over Rician multipath fading channels with different number of users ( $\mathrm{K}=10,20$ and 30$)$ using Gold codes $(\mathrm{N}=127)$.

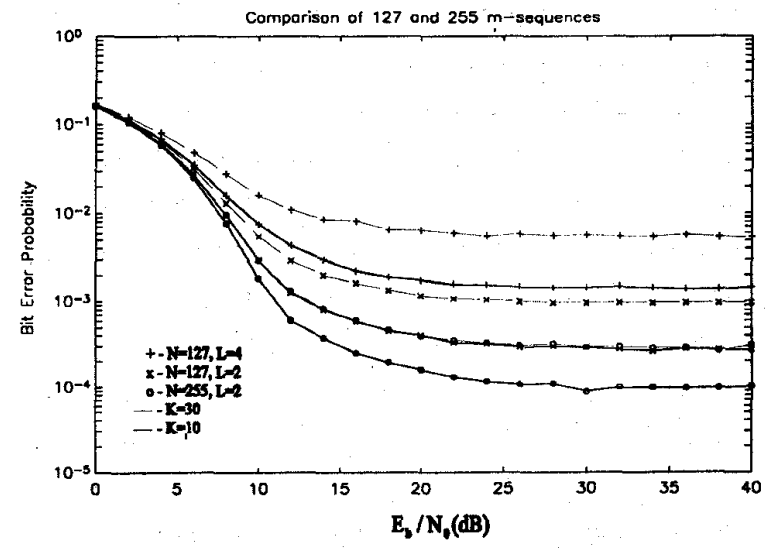

Figure 4. Comparison of the performance of the CPA CDMA system over Rician multipath fading channels with different number of users $(K=10,30)$, different number of resolvable paths $(\mathrm{L}=2$, 